\title{
Fasting blood glucose levels and prognosis in patients with non-small-cell lung cancer: a prospective cohort study in China
}

This article was published in the following Dove Press journal: OncoTargets and Therapy

Jin-Rong Yang,' Guo-Chong Chen,' Jia-Ying $X u,{ }^{2}$ Chen-Jie Ling, ${ }^{1} \mathrm{Na} \mathrm{Yu}{ }^{3}$ Jing Yang, ${ }^{4} \mathrm{Da}-X i o n g$ Zeng, ${ }^{5}$ MinJing Gu, ${ }^{6}$ Da-Peng Li, ${ }^{6}$ YuSong Zhang, ${ }^{3}$ Li-Qiang Qin ${ }^{1}$

'Department of Nutrition and Food Hygiene, School of Public Health, Soochow University, Suzhou 215I23, People's Republic of China; ${ }^{2}$ Institute of Quantitative Biology and Medicine, State Key Laboratory of Radiation Medicine and Protection,

School of Radiation Medicine and Protection, Soochow University, Suzhou 215I23, People's Republic of China; ${ }^{3}$ Department of Oncology, The Second Affiliated Hospital of Soochow University, Suzhou 215004, People's Republic of China; ${ }^{4}$ Department of Clinical Nutrition, The First Affiliated Hospital of Soochow University, Suzhou 2I 503I, People's Republic of China; ${ }^{5}$ Department of Respiration, The First Affiliated Hospital of Soochow University, Suzhou 21503I, People's Republic of China; ${ }^{6}$ Department of Oncology, The First Affiliated Hospital of Soochow University, Suzhou 2 I503I, People's Republic of China

Correspondence: Li-Qiang Qin

Department of Nutrition and Food Hygiene, School of Public Health, Soochow University, 199 Renai Road, Suzhou 215123, People's Republic of China Tel +865 I26588 007|

Email qinliqiang@suda.edu.cn

Yu-Song Zhang

Department of Oncology, The Second

Affiliated Hospital of Soochow University,

1055 Sanxiang Road, Suzhou 215004,

People's Republic of China

Tel +86 5 I 267784286

Email zhangyusong19@163.com
Purpose: Non-small-cell lung cancer (NSCLC) is the most diagnosed lung cancer and is associated with poor prognosis. This study aimed to analyze whether fasting blood glucose (FBG) levels could provide prognostic information in Chinese patients with NSCLC, using the Suzhou Lung Cancer Survival study.

Patients and methods: A prospective cohort study of adult patients with primary NSCLC was performed. The patients who were hospitalized between January 2016 and April 2018 in two hospitals affiliated with Soochow University were recruited. Patient information, including lifestyle habits and clinical and laboratory data, were collected through face-to-face interviews and evaluation of medical records. Follow-up was initiated from the date of patient enrollment until May 8, 2018 or until patient death. The long-term survival of patients was assessed every 6 months. Patient vital status was confirmed by using hospital records, telephone interview, or local death registration system. Cox proportional hazards regression was used to estimate hazard ratio and $95 \%$ confidence interval (CI) for death, with adjustment for cancer stage, medical treatments, smoking, and other potential confounders.

Results: A total of 387 patients were included in the analysis, and the numbers (percentages) of patients with stages I, II, III, and IV NSCLC were 53 (13.7\%), 41 (10.6\%), 64 (16.5\%), and $215(55.6 \%)$, respectively. The median duration of follow-up was 19.1 months. Compared with patients in the second tertile of FBG, the HRs for mortality were 2.16 (95\% CI: 1.26-3.73) and 1.87 (95\% CI: 1.03-3.42) for those in the lowest one and diabetic group, respectively. Subgroup analysis according to various patient characteristics confirmed these associations.

Conclusion: Diabetes and low FBG could be important predictors of death in patients with NSCLC. Maintaining appropriate blood glucose levels may improve prognosis in patients with NSCLC.

Keywords: non-small cell lung cancer, fasting blood glucose, diabetes, survival

\section{Introduction}

Globally, lung cancer is one of the most common causes of cancer-related death and is associated with poor treatment outcome. ${ }^{1}$ Non-small-cell lung cancer (NSCLC) is the most common type of lung cancer, accounting for $>80 \%$ of all lung cancer cases. ${ }^{2}$ The majority of patients with NSCLC are usually diagnosed at later stages when curative treatments are unavailable. In this regard, identifying prognostic factors is important because these factors could improve clinical decision making and, ultimately, patient prognosis. A number of prognostic factors in NSCLC have been described. In a systematic review of literature published between 1990 and 2001, 
Brundage et al, found 169 potential prognostic factors that may be useful in predicting the survival of patients with NSCLC. ${ }^{3}$ However, most of these factors are not readily available in routine practice.

The presence of multiple diseases is common among lung cancer patients. Notably, $8-18 \%$ of newly diagnosed cancer patients have diabetes. Although diabetes is the most common comorbidity associated with poor cancer survival, ${ }^{4}$ the influence of diabetes on NSCLC prognosis remains equivocal. ${ }^{5}$ Several observational studies found that diabetes was associated with poor prognosis in patients with NSCLC, ${ }^{6-9}$ whereas other studies showed that diabetes was related to equal ${ }^{10}$ or better survival. ${ }^{11,12}$ Diabetes may affect lung cancer progression via several mechanisms, such as hyperinsulinemia, hyperglycemia, and chronic inflammation, which are associated with cell proliferation and cancer progression. ${ }^{13,14}$ On the contrary, diabetes also impedes cancer cell invasion through the basocellular membrane by diabetic microangiopathy via microvascular changes, resulting in decreased metastatic ability and improved prognosis. ${ }^{15}$ Thus far, only a few studies investigated the prognostic impact of fasting blood glucose (FBG) on NSCLC mortality and found that hyperglycemia may have significantly lower survival. ${ }^{16,17}$ However, blood glucose has not been observed in a further stratification. Therefore, we used the data from the Suzhou Lung Cancer Survival (SLCS) study to investigate whether FBG in the low or high range could provide prognostic information in patients with NSCLC.

\section{Materials and methods}

\section{Participant identification}

The SLCS study is an ongoing patient cohort that was initiated in 2015 to explore the long-term predictors of lung cancer survival among patients living in Suzhou, China. Primary lung cancer patients aged 18 years old or older were included in the study. Although our inclusion criteria did not have other specific restrictions, such as status and type of medical treatments, data were carefully collected during the enrollment period and their influence on any examined association were explored through rigorous analysis. The patient enrollment was started in January 2016. In April 2018, a total of 455 patients with primary lung cancer were included. Considering the small number of small cell lung cancer cases $(n=49)$, our present analysis only included patients with NSCLC $(n=406)$. This study was approved by the Research Ethics Committee of Soochow University (Approval No. ESCU-2015-0002) and written informed consent was obtained from each patient. This study was also conducted in accordance with the Declaration of Helsinki.

\section{Data collection}

In-person interviews were conducted using a structured questionnaire during recruitment to collect patients' characteristics on demographics and habitual lifestyle including smoking, alcohol drinking, and physical activity. Based on the collected data, smoking status was grouped into three categories (never, former, or current smoker) and drinking status was classified into two groups (yes or no). Former smokers were defined as patients who had smoked but already quitted smoking for at least one year, and current smokers were determined as those who are still smoking at the time of investigation or those who gave up smoking for less than one year. Patients who are drinking alcohol on average of at least once a week within the previous six months were considered as patients with drinking habit (yes). Anthropometric measurements (height, weight, waist, and hip circumference) were measured by trained personnel. The pathological type, cancer stage, and the treatments for lung cancer were identified through medical records.

\section{Blood sample collection}

A $5 \mathrm{~mL}$ whole blood was drawn from antecubital veins of each patient and then transferred into a tube with ethylenediaminetetraacetic acid during recruitment after an overnight fasting. The plasma was obtained through centrifugation (3000 rpm at $25^{\circ} \mathrm{C}$ for $10 \mathrm{mins}$ ) within $2 \mathrm{hrs}$ after sample collection. All samples were numbered and stored at $-80^{\circ} \mathrm{C}$ until further analysis.

\section{Measurements of biomarkers}

Measurement of FBG and albumin was conducted in laboratories of both institutions as a part of routine examination in each patient. FBG levels were measured by the hexokinase method using a fully automatic Siemens ADVIA 2400 Biochemical Analyzer System (Siemens Healthcare Diagnosis Inc, NY, USA). Albumin concentration was measured by bromocresol green staining method with an Olympus 5400 Automatic Biochemical Analyzer (Olympus Corporation, Tokyo, Japan).

\section{Patient follow-up}

This study primarily determined the overall survival, which was calculated from the date of the baseline 
questionnaire to the date of death from any cause. Followup was initiated from the date of patient enrollment until May 8, 2018 or until patient death. The long-term survival of patients was assessed every 6 months. Data were collected from at least one of the following sources: 1) the hospital inpatient or outpatient records; 2) local death registration system; and 3) contacting patients or their family on phones. A small proportion of patients $(4.7 \%$, $\mathrm{n}=19$ ) were excluded from the present analysis due to loss of follow-up. Finally, a total of 387 patients with NSCLC were included in the present analysis.

\section{Statistical analysis}

Continuous variables were expressed as the means with the standard deviation and were compared using one-way ANOVA. The Wilcoxon rank test was used to compare the categorical variables, which were presented as the number and percentage of patients. Cases with diabetes, information on treatment for diabetes, or FBG value of or higher than $126 \mathrm{mg} /$ $\mathrm{dL}$ were included in a separate category (diabetic). For nondiabetic cases, FBG levels were categorized according to the following tertiles: $<91,91-101$ (reference), and $>101 \mathrm{mg} / \mathrm{dL}$.

The Hazard ratios (HR) and 95\% confidence intervals (CI) for death were estimated using the Cox proportional hazards regression model. Two models with different covariates were constructed to account for potential confounders. The first model was adjusted for patient demographic and behavioral characteristics, including age at baseline interview (years), sex, body mass index $\left(\mathrm{kg} / \mathrm{m}^{2}\right)$, albumin ( $<35 \mathrm{~g} / \mathrm{L}$ or $\geq 35 \mathrm{~g} / \mathrm{L}$ ), family history of cancer (yes or no), patient history of chronic obstructive pulmonary disease (yes or no), smoking status (never smoker, former smoker, or current smoker), and drinking habit (yes or no). The second model included the variables in model 1 plus additional adjustment for clinical and pathological information, including tumor type (adenocarcinoma, squamous cell carcinoma, or other tumor types), cancer stage (I/II or III/IV), and treatment (with tumor resection, without resection but with other therapies such as chemotherapy and radiotherapy, without either resection or other therapies).

We also performed stratified analyses according to age ( $<60$ years vs $\geq 60$ years), sex, smoking status (never smoker vs ever smoker), tumor type (adenocarcinoma vs squamous cell carcinoma), and cancer stage (I-III vs IV) to assess the potential effect modification by these characteristics. Statistical analyses were performed using the SAS 9.2 software. All tests were two-sided and statistical significance was set at $P<0.05$.

\section{Results}

A total of 387 patients with NSCLC were included in the final analysis. Mean age of the participants was 62.5 years. Two hundred and forty-one patients were male (62.3\%), and the majority of them were at an advanced stage (72.1\%). The median duration of follow-up was 19.1 months. Overall, 105 patients died by the end of May 2018. The baseline characteristics of the patients by FBG level are presented in Table 1. FBG levels were significantly associated with body mass index.

Results of multivariate analysis with adjustment for confounders are presented in Table 2. After adjustment of age, sex, body mass index, family history of cancer, patient history of chronic obstructive pulmonary disease, smoking status and drinking habit, albumin, the risk of death was significantly higher for those with the lowest tertile of blood glucose (HR $=2.03$; 95\% CI: 1.19-3.46) and diabetic group ( $\mathrm{HR}=1.81$; 95\% CI: 1.01-3.26), compared with those in the reference. Further adjustment for other clinical and pathological factors, such as tumor type, cancer stage, and treatment showed similar results. The HRs for mortality were 2.16 (95\% CI: 1.26-3.73) and 1.87 (95\% CI: $1.03-3.42)$ for those in the lowest tertile of FBG and diabetic group, respectively.

Results of subgroup analysis according to various patient characteristics confirmed these associations (Table 3). FBG levels of lower than $91 \mathrm{mg} / \mathrm{dL}$ or within a diabetic group were associated with a higher risk of death. Notably, the association between FBG and death evidently varied with tumor type and cancer stage. For patients with squamous cell carcinoma, FBG levels of lower than $91 \mathrm{mg} / \mathrm{dL}$ were not associated with an increased risk of mortality. Patients with diabetic status had no association with a higher risk of death in subgroup of stage I/II/III.

\section{Discussion}

The present study identified that FBG levels of lower than $91 \mathrm{mg} / \mathrm{dL}$ or within diabetic range were associated with poor survival in Chinese NSCLC patients. These associations remained robust even after adjustment for other potential confounders. Subgroup analysis according to various patient characteristics also confirmed these associations.

Several previous studies that classified patients according to the presence or absence of diabetes showed that diabetes was associated with poorer survival in patients with NSCLC. ${ }^{6-9}$ The current study demonstrated that NSCLC patients with diabetes exhibited a significant increase in mortality risk, 
Table I Baseline characteristics of patients by fasting blood glucose level

\begin{tabular}{|c|c|c|c|c|c|}
\hline & \multicolumn{4}{|c|}{ Fasting blood glucose (mg/dL) } & \multirow[b]{2}{*}{$P$-value } \\
\hline & $<91$ & $91-101$ & $>101$ & diabetic & \\
\hline Age & $62.43 \pm 10.23$ & $60.46 \pm 11.74$ & $64.43 \pm 9.05$ & $62.80 \pm 9.94$ & 0.056 \\
\hline \multicolumn{6}{|l|}{ Gender } \\
\hline Male & $66(62.3)$ & $66(57.4)$ & $62(67.4)$ & $47(63.5)$ & 0.522 \\
\hline Female & $40(37.7)$ & $49(42.6)$ & $30(32.6)$ & $27(36.5)$ & \\
\hline \multicolumn{6}{|l|}{ Smoking status } \\
\hline Never & $49(46.2)$ & $63(54.8)$ & $40(43.5)$ & $31(4 I .9)$ & 0.124 \\
\hline Former & $26(24.5)$ & $22(19.1)$ & $27(29.3)$ & $27(36.5)$ & \\
\hline Current & $31(29.3)$ & $30(26.1)$ & $25(27.2)$ & $16(21.6)$ & \\
\hline \multicolumn{6}{|l|}{ Tumor type } \\
\hline$A C$ & $70(66.0)$ & $81(70.4)$ & $64(69.6)$ & $44(59.5)$ & 0.773 \\
\hline SCC & $29(27.4)$ & $23(20.0)$ & $21(22.8)$ & $23(31.1)$ & \\
\hline Other & $7(6.6)$ & II (9.6) & $7(7.6)$ & $7(9.5)$ & \\
\hline \multicolumn{6}{|l|}{ Cancer stage } \\
\hline Stage I/II/III & $39(36.8)$ & $50(43.5)$ & $39(42.4)$ & $30(40.5)$ & 0.458 \\
\hline Stage IV & $64(60.4)$ & $60(52.2)$ & $52(56.5)$ & $39(52.7)$ & \\
\hline Unknown & $3(2.8)$ & $5(4.3)$ & I (I.I) & $5(6.8)$ & \\
\hline \multicolumn{6}{|l|}{ Treatment } \\
\hline Surgery & 38 (35.9) & $60(52.1)$ & $39(42.4)$ & $32(43.2)$ & 0.697 \\
\hline Other therapy & $53(50.0)$ & $47(40.9)$ & 44 (47.8) & $34(46.0)$ & \\
\hline None & $15(14.1)$ & $8(7.0)$ & $9(9.8)$ & $8(10.8)$ & \\
\hline \multicolumn{6}{|l|}{ Albumin } \\
\hline$<35 \mathrm{~g} / \mathrm{L}$ & $21(19.8)$ & I3 (II.3) & $4(4.4)$ & $16(21.6)$ & 0.462 \\
\hline$\geq 35 \mathrm{~g} / \mathrm{L}$ & $85(80.2)$ & $102(88.7)$ & $88(95.6)$ & $58(78.4)$ & \\
\hline \multicolumn{6}{|l|}{ BMI } \\
\hline$<18.5$ & $16(15.1)$ & $12(10.4)$ & $6(6.5)$ & $3(4.0)$ & 0.007 \\
\hline $18.5-23.9$ & $65(81.3)$ & $7 \mid(6 I .7)$ & $52(56.5)$ & $49(66.2)$ & \\
\hline $24.0-27.9$ & $17(16.0)$ & $24(20.9)$ & $30(32.6)$ & $19(25.7)$ & \\
\hline$\geq 28.0$ & $8(7.6)$ & $8(7.0)$ & $4(4.4)$ & $3(4.1)$ & \\
\hline
\end{tabular}

Abbreviations: AC, adenocarcinoma; BMI, body mass index; SCC, squamous cell carcinoma.

Table 2 COX proportional hazards regression overall model of death and fasting blood glucose

\begin{tabular}{|l|l|l|l|l|l|}
\hline \multirow{2}{*}{ FBG (mg/dL) } & \multirow{2}{*}{$\mathbf{N}$} & \multicolumn{2}{l|}{ Model I } & \multicolumn{2}{l|}{ Model 2 } \\
\cline { 3 - 6 } & & HR (95\% Cl) & P-value & \multicolumn{2}{l|}{ HR (95\% CI) } \\
\hline$<91$ & 106 & $2.03(1.19-3.46)$ & 0.009 & $2.16(1.26-3.73)$ & P-value \\
$91-101$ & 115 & 1 & & 1 & 0.006 \\
$>101$ & 92 & $1.33(0.74-2.4 I)$ & 0.34 & $1.54(0.85-2.80)$ & 0.159 \\
Diabetic & 74 & $1.81(1.01-3.26)$ & 0.049 & $1.87(1.03-3.42)$ & 0.041 \\
\hline
\end{tabular}

Notes: Model I includes age at baseline interview, sex, body mass index, family history of cancer, patient history of chronic obstructive pulmonary disease, smoking status, drinking habit, and albumin. Model 2: model I plus tumor type, cancer stage, and treatment.

Abbreviations: $\mathrm{Cl}$, confidence interval; $\mathrm{FBG}$, fasting blood glucose; HR, hazard ratio.

which is consistent with a meta-analysis showing that the association of diabetes with inferior prognosis was more prominent in the NSCLC group than that in the small-cell lung cancer group. ${ }^{18}$ Two studies indicated that the risk of death increased with the increment of FBG without consideration of diabetic status in gastric carcinoma ${ }^{19}$ or esophageal squamous 
Table 3 COX proportional hazards regression stratified models of death and fasting blood glucose

\begin{tabular}{|c|c|c|c|c|c|}
\hline & \multirow[t]{3}{*}{ Death/total } & \multicolumn{4}{|c|}{ Hazard ratio ( $95 \%$ confidence interval) } \\
\hline & & \multicolumn{4}{|c|}{ Fasting blood glucose $(\mathrm{mg} / \mathrm{dL})$} \\
\hline & & $<91$ & $91-101$ & $>10 \mid$ & Diabetic \\
\hline \multicolumn{6}{|l|}{ Age } \\
\hline$<60$ & $29 / 138$ & $3.75(1.13-12.49)$ & I & $2.10(0.54-8.26)$ & $1.62(0.44-5.93)$ \\
\hline$\geq 60$ & $76 / 249$ & $1.78(0.92-3.44)$ & I & $\mathrm{I} .43(0.7 \mathrm{I}-2.87)$ & $2.04(1.00-4.14)$ \\
\hline \multicolumn{6}{|l|}{ Gender } \\
\hline Male & $79 / 241$ & $1.73(0.94-3.18)$ & I & $1.56(0.80-3.04)$ & $1.50(0.72-3.11)$ \\
\hline Female & $26 / 146$ & $4.96(1.31-18.76)$ & I & $1.50(0.36-6.27)$ & $3.38(0.91-12.52)$ \\
\hline \multicolumn{6}{|l|}{ Smoking } \\
\hline Never & $35 / 183$ & $4.31(1.46-12.75)$ & I & $1.66(0.52-5.35)$ & $3.88(1.28-11.73)$ \\
\hline Ever & $70 / 204$ & $1.61(0.84-3.10)$ & I & $1.57(0.75-3.28)$ & $1.26(0.57-2.77)$ \\
\hline \multicolumn{6}{|c|}{ Tumor type } \\
\hline$A C$ & $58 / 259$ & $3.49(1.55-7.90)$ & I & $2.25(0.97-5.22)$ & $3.01(1.18-7.66)$ \\
\hline SCC & $35 / 96$ & $0.83(0.82-2.13)$ & I & $0.7 I(0.2 I-2.4 I)$ & $1.32(0.46-3.8 I)$ \\
\hline \multicolumn{6}{|c|}{ Cancer stage } \\
\hline$|/| I / / I I I$ & $26 / 158$ & $1.56(0.5 \mathrm{I}-4.79)$ & I & $0.69(0.20-2.34)$ & $0.89(0.27-2.34)$ \\
\hline IV & $76 / 215$ & $\mathrm{I} .96(\mathrm{I} .0 \mathrm{I}-3.78)$ & I & $1.34(0.66-2.75)$ & $2.11(0.99-4.46)$ \\
\hline
\end{tabular}

Notes: Exclude stratification factor when the model was running. Model includes age at baseline interview, sex, body mass index, family history of cancer, patient history of chronic obstructive pulmonary disease, smoking status, drinking habit, albumin, tumor type, cancer stage, and treatment.

Abbreviations: AC, adenocarcinoma; SCC, squamous cell carcinoma.

cell carcinoma patients. ${ }^{20}$ Park et al, found positive linear trends in lung cancer mortality with increasing fasting serum glucose in Korean patients, especially in patients with fasting serum level of $126 \mathrm{mg} / \mathrm{dL}$ or higher. ${ }^{21}$ The biological mechanisms underlying the increased mortality risk in patients with NSCLC remain unclear. Diabetes is characterized by insulin resistance and hyperinsulinemia. ${ }^{22}$ Experimental studies found that most cancer cells express insulin receptors, and hyperinsulinemia promotes carcinogenesis by directly stimulating the proliferating pathway after insulin receptors. ${ }^{23}$ Cancer patients with diabetes may increase tumor cell proliferation and metastases as a consequence of the mitogenic environment of hyperinsulinemia. ${ }^{24}$

The present study identified that FBG levels lower than $91 \mathrm{mg} / \mathrm{dL}$ were associated with poor survival in patients with NSCLC. The reason for adverse effect of low blood glucose levels on NSCLC prognosis is unclear. In general, low FBG was significantly associated with malnutrition of patients with NSCLC and hence resulting poor survival in patients with NSCLC. ${ }^{25}$ There is evidence that FBG lower than 90 $\mathrm{mg} / \mathrm{dL}$ was associated with a reduced survival in esophageal cancer patient. ${ }^{25}$ Minicozzi et al, also showed that breast cancer patients with FBG levels of $84 \mathrm{mg} / \mathrm{dL}$ or lower had a non-significant higher risk of death. ${ }^{26}$ Further studies are needed to investigate the underlying mechanisms by which low FBG levels increase mortality risk in NSCLC patients.

The present study had several limitations. First, although a wide range of patient characteristics was considered in the analysis, the influence of unmeasured and residual confounders that may bias the observed findings could not be fully ruled out. Second, small number of patients included in the subgroup analyses may limit statistical power to detect a weak association. For example, no association was found when the results were further stratified by types of squamous cell carcinoma and stages of cancer. Ongoing patient enrollment could provide further opportunity to specifically investigate some of these associations. Third, the findings of the present study may not be applied to the general population because only patients in two hospitals were included.

\section{Conclusion}

In summary, the present study showed that low FBG levels and diabetes were associated with poor survival in Chinese patients 
with NSCLC. Additional studies are still needed to verify the relationship between FBG levels and survival among patients with NSCLC.

\section{Abbreviation list}

$\mathrm{AC}$, adenocarcinoma; BMI, body mass index; CI, confidence interval; FBG, fasting blood glucose; HR, hazard ratio; NSCLC, non-small cell lung cancer; SCC, squamous cell carcinoma; SLCS, Suzhou Lung Cancer Survival.

\section{Acknowledgments}

This work was supported by the National Natural Science Foundation of China (No. 81472974, 81673101) and the Priority Academic Program Development of Jiangsu Higher Education Institutions (PAPD).

\section{Disclosure}

The authors report no conflicts of interest in this work.

\section{References}

1. Bray F, Ferlay J, Soerjomataram I, Siegel RL, Torre LA, Jemal A. Global cancer statistics 2018: GLOBOCAN estimates of incidence and mortality worldwide for 36 cancers in 185 countries. CA Cancer $J$ Clin. 2018;68(6):394-424. doi:10.3322/caac.21492

2. Ettinger DS, Wood DE, Aisner DL, et al. Non-small cell lung cancer, version 5.2017, NCCN clinical practice guidelines in oncology. $J$ Natl Compr Canc Netw. 2017;15(4):504-535.

3. Brundage MD, Davies D, Mackillop WJ. Prognostic factors in nonsmall cell lung cancer: a decade of progress. Chest. 2002;122(3):10371057. doi:10.1378/chest.122.3.1037

4. Tammemagi CM, Neslund-Dudas C, Simoff M, Kvale P. Impact of comorbidity on lung cancer survival. Int J Cancer. 2003;103(6):792802. doi:10.1002/ijc. 10882

5. Barone BB, Yeh HC, Snyder CF, et al. Long-term all-cause mortality in cancer patients with preexisting diabetes mellitus: a systematic review and meta-analysis. Jama. 2008;300(23):2754-2764. doi:10.1001/jama.2008.824

6. Inal A, Kaplan MA, Kucukoner M, Urakci Z, Kilinc F, Isikdogan A. Is diabetes mellitus a negative prognostic factor for the treatment of advanced non-small-cell lung cancer? Rev Port Pneumol. 2014;20 (2):62-68. doi:10.1016/j.rppneu.2013.09.001

7. van de Poll-Franse LV, Houterman S, Janssen-Heijnen ML, Dercksen MW, Coebergh JW, Haak HR. Less aggressive treatment and worse overall survival in cancer patients with diabetes: a large population based analysis. Int J Cancer. 2007;120(9):1986-1992. doi:10.1002/ijc.22532

8. Imai H, Kaira K, Mori K, et al. Prognostic significance of diabetes mellitus in locally advanced non-small cell lung cancer. BMC Cancer. 2015;15:989. doi:10.1186/s12885-015-1584-3

9. Win T, Sharples L, Groves AM, Ritchie AJ, Wells FC, Laroche CM. Predicting survival in potentially curable lung cancer patients. Lung. 2008;186(2):97-102. doi:10.1007/s00408-007-9067-1
10. Nakazawa K, Kurishima K, Tamura T, Ishikawa H, Satoh H, Hizawa N. Survival difference in NSCLC and SCLC patients with diabetes mellitus according to the first-line therapy. Med Oncol. 2013;30 (1):367. doi:10.1007/s12032-012-0367-9

11. Hanbali A, Al-Khasawneh K, Cole-Johnson C, Divine G, Ali H. Protective effect of diabetes against metastasis in patients with nonsmall cell lung cancer. Arch Intern Med. 2007;167(5):513.

12. Bartling B, Simm A, Sohst A, Silber RE, Hofmann HS. Effect of diabetes mellitus on the outcome of patients with resected non-small cell lung carcinoma. Gerontology. 2011;57(6):497-501. doi:10.1159/000323856

13. Morss AS, Edelman ER. Glucose modulates basement membrane fibroblast growth factor-2 via alterations in endothelial cell permeability. J Biol Chem. 2007;282(19):14635-14644. doi:10.1074/jbc.M608565200

14. Garcia-Jimenez C, Garcia-Martinez JM, Chocarro-Calvo A, De la Vieja A. A new link between diabetes and cancer: enhanced WNT/ beta-catenin signaling by high glucose. $J$ Mol Endocrinol. 2014;52 (1):R51-R66. doi:10.1530/JME-13-0152

15. Nerlich AG, Hagedorn HG, Boheim M, Schleicher ED. Patients with diabetes-induced microangiopathy show a reduced frequency of carcinomas. In Vivo. 1998;12(6):667-670.

16. Luo J, Chen YJ, Chang LJ. Fasting blood glucose level and prognosis in non-small cell lung cancer (NSCLC) patients. Lung Cancer. 2012;76(2):242-247. doi:10.1016/j.lungcan.2011.10.019

17. Kirakli EK, Yilmaz U, Yilmaz H, Komurcuoglu B. Fasting blood glucose level in locally advanced non-small cell lung cancer: a new prognostic factor? Horm Cancer. 2018;9(3):188-196. doi:10.1007/s12672-0180322-0

18. Zhu L, Cao H, Zhang T, et al. The effect of diabetes mellitus on lung cancer prognosis: a prisma-compliant meta-analysis of cohort studies. Medicine. 2016;95(17):e3528. doi:10.1097/MD.0000000000004864

19. Lin Y, Hu D, Zhou Q, Lin X, Lin J, Peng F. The fasting blood glucose and long non-coding RNA SNHG8 predict poor prognosis in patients with gastric carcinoma after radical gastrectomy. Aging (Albany NY). 2018;10 (10):2646-2656. doi:10.18632/aging.101576

20. Hu D, Peng F, Lin X, et al. The elevated preoperative fasting blood glucose predicts a poor prognosis in patients with esophageal squamous cell carcinoma: the Fujian prospective investigation of cancer (FIESTA) study. Oncotarget. 2016;7(40):65247-65256. doi:10.18632/ oncotarget. 11247

21. Park SM, Lim MK, Shin SA, Yun YH. Impact of prediagnosis smoking, alcohol, obesity, and insulin resistance on survival in male cancer patients: national health insurance corporation study. J Clin Oncol. 2006;24(31):5017-5024. doi:10.1200/JCO.2006.07.0243

22. Jee SH, Ohrr H, Sull JW, Yun JE, Ji M, Samet JM. Fasting serum glucose level and cancer risk in Korean men and women. Jama. 2005;293(2):194-202. doi:10.1001/jama.293.2.194

23. Giovannucci E, Harlan DM, Archer MC, et al. Diabetes and cancer: a consensus report. Diabetes Care. 2010;33(7):1674-1685. doi:10.2337/ dc10-0666

24. Richardson LC, Pollack LA. Therapy insight: influence of type 2 diabetes on the development, treatment and outcomes of cancer. Nat Clin Pract Oncol. 2005;2(1):48-53. doi:10.1038/ncponc0062

25. Wu N, Zhu Y, Kadel D, Pang L, Chen G, Chen Z. The prognostic influence of body mass index, resting energy expenditure and fasting blood glucose on postoperative patients with esophageal cancer. BMC Gastroenterol. 2016;16(1):142. doi:10.1186/s12876016-0549-6

26. Minicozzi P, Berrino F, Sebastiani F, et al. High fasting blood glucose and obesity significantly and independently increase risk of breast cancer death in hormone receptor-positive disease. Eur $J$ Cancer. 2013;49(18):3881-3888. doi:10.1016/j.ejca.2013.08.004 


\section{Publish your work in this journal}

OncoTargets and Therapy is an international, peer-reviewed, open access journal focusing on the pathological basis of all cancers, potential targets for therapy and treatment protocols employed to improve the management of cancer patients. The journal also focuses on the impact of management programs and new therapeutic agents and protocols on patient perspectives such as quality of life, adherence and satisfaction. The manuscript management system is completely online and includes a very quick and fair peer-review system, which is all easy to use. Visit http://www.dovepress.com/ testimonials.php to read real quotes from published authors.

Submit your manuscript here: https://www.dovepress.com/oncotargets-and-therapy-journal 